\title{
CALCIUM AND PHOSPHORUS METABOLISM IN OSTEOMALACIA $X$. FURTHER STUDIES ON VITAMIN D ACTION : EARLY SIGNS OF DEPLETION AND EFFECT OF MINIMAL DOSES
}

\author{
By H. I. CHU, S. H. LIU, T. F. YU, H. C. HSU, T. Y. CHENG ANd H. C. CHAO \\ (From the Department of Medicine, Peiping Union Medical College, Peiping, China)
}

(Received for publication October 24, 1939)

Hannon and associates (9) in the first communication of this series demonstrated the prolonged beneficial effect of vitamin D on the calcium and phosphorus metabolism in osteomalacia. A daily dose of 7,500 international units of vita$\min \mathrm{D}$ in the form of Vigantol (Bayer) administered orally for a period of sixteen days in a case of osteomalacia brought about a marked calcium and phosphorus retention which continued for more than three months after the drug was discontinued. Since the beneficial effect continued unabated even at the end of observation, the actual duration of this action must be longer than three or four months. Similar results were obtained when a series of brief ultraviolet irradiations from a mercury vapor quartz lamp was applied in place of oral administration of vitamin D (3).

In attempting to explain the prolonged action of vitamin $\mathrm{D}$, two possibilities were considered. First, the vitamin D may be stored. This was dismissed at the time, because of lack of knowledge on vitamin $\mathrm{D}$ storage. The second possibility, which was considered the more likely explanation, is related to the vitamin D content of the diets served to the patient. The minimal amounts of vitamin D that may be present in the diets, though insufficient to bring about the reparative process, may suffice to maintain normal calcium and phosphorus metabolism as soon as the initial deficiency is corrected by vitamin D or ultraviolet irradiation therapy.

The present communication is concerned with (1) observation on the antirachitic potency of the vitamin $\mathrm{D}$-deficient diets commonly used in our investigation of calcium and phosphorus metabolism in osteomalacia, (2) the effect of a minimal dose of vitamin $\mathrm{D}$ and (3) the changes of calcium and phosphorus metabolism following the withdrawal of vitamin $D$.

\section{PROCEDURE}

Three cases of osteomalacia and one normal newborn baby from an osteomalacic mother were studied in the present investigation. Their clinical histories are briefly described in the appendix. Data on Cases 3 and 4 were published previously in other connections $(4,15)$. They were kept in the metabolism ward throughout the period of study and quantitative, vitamin D-deficient and low calcium diets were served. The composition of these diets is shown in Table I. Calcium was administered in the form of a 7.7 per cent solution of calcium lactate in Cases 1 and 2. The infant, Case 4, was fed exclusively on measured quantities of his mother's milk. Calcium, phosphorus and nitrogen balances were determined in four-day periods continuously in Cases 1 to 3 , but in Case 4, 2 four-day periods of study were alternated with 2 periods without metabolic observations. The organization of the metabolism ward, the preparation of quantitative diets, the collection of excreta and the methods of chemical analysis of food and milk, stool and urine, and serum were described in our early publication (16).

Case 1 was a young woman with osteomalacia who was convalescing from an obstetrical operation and pulmonary tuberculosis in the obstetric service for nearly a month before she was transferred to the metabolism ward. She was put on the basal vitamin D-deficient diet for a long time under metabolic observation just to ascertain the antirachitic potency of the diet. Then acid and alkali salt mixtures of Shohl (17) were given for the purpose of studying the acid and base metabolism which we are not concerned with in the present communication. Then minimal doses of vitamin $D$ in the form of Vigantol and egg were administered on separate occasions to observe their effect on calcium and phosphorus metabolism, particularly the changes following their withdrawal. Case 2 was similarly studied except that prolonged control observation on the basal diet alone was not made. Case 3 was studied primarily from the standpoint of the effect of acid and alkali on the calcium and phosphorus metabolism but the data were reproduced here because they illustrate two of the three propositions in which we are interested in the present communication. Calcium, phosphorus and nitrogen metabolism was observed in Case 4 until what we consider the incipient changes of vitamin D-deficiency appeared. Then the reverse processes were studied after vitamin $D$ was given to the lactating mother. 
TABLE I

Composition of diets*

(Grams per day)

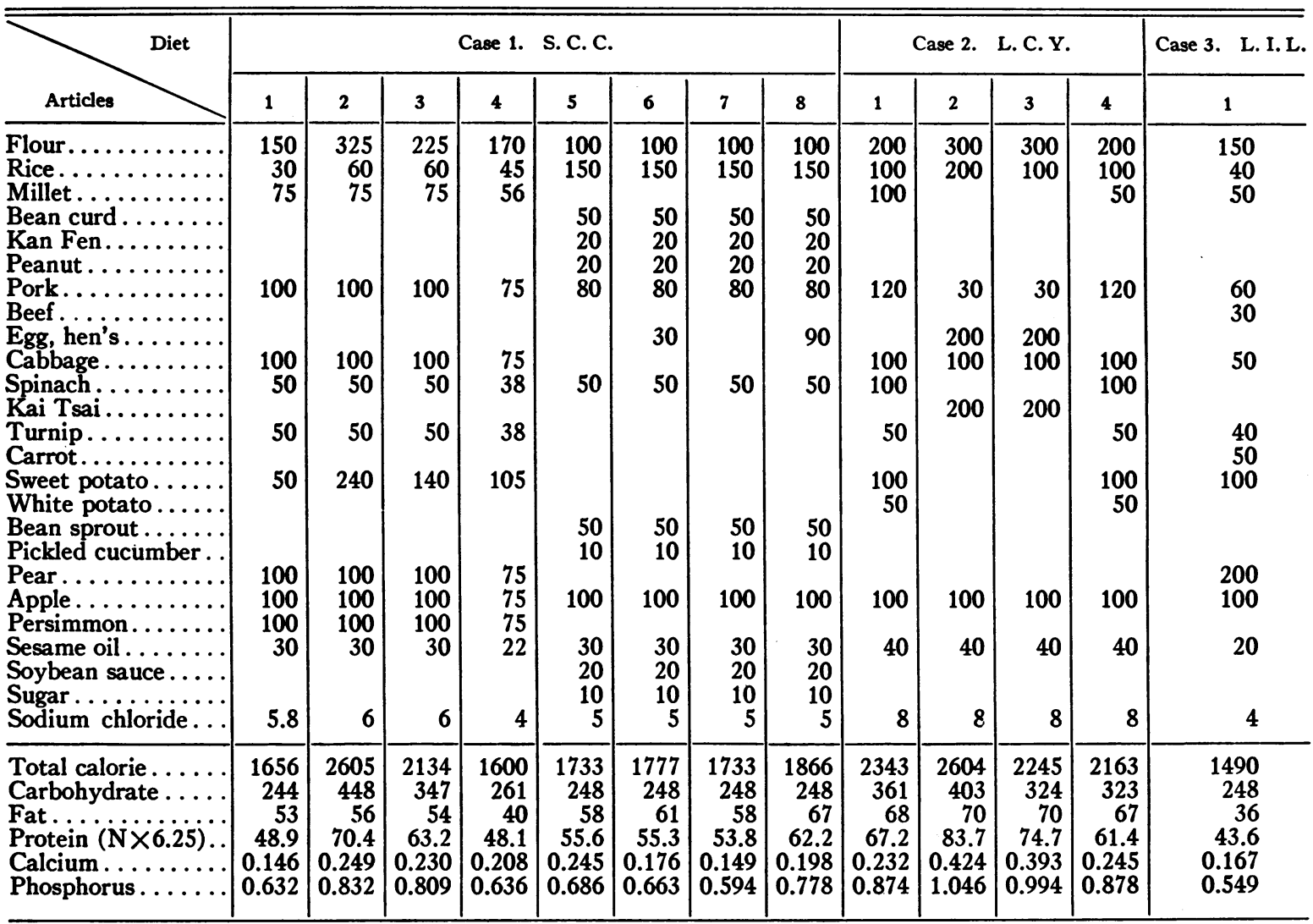

* Calorie, carbohydrate and fat are calculated values (Outlines of Diets of the Peiping Union Medical College Hospital, Peiping, 1937, 3rd edition). Protein, calcium and phosphorus are analyzed values.

\section{RESULTS}

Case 1, S. C. C., osteomalacia

I. The antirachitic potency of the previous dietary and the lack of such potency in the experimental diets with resulting vitamin $D$ depletion. Data obtained in periods 1 to 12 (Table II and Figure 1) indicate that, when the patient was first under metabolic observation on the experimental vitamin $\mathrm{D}$-deficient diets, a good calcium and phosphorus balance was obtained. The calcium retention in periods 1 to 4 amounted to an average of 54 per cent of the intake and the phosphorus retention in the same periods, 58 per cent. Urinary phosphorus became almost nil in period 4, while considerable amounts of calcium appeared in the urine in periods 3 to 4 . The disappearance of urinary phosphorus was probably in part related to the exceptionally high retention of nitro- gen in those periods which required an equivalent amount of phosphorus for deposition as soft tissues, leaving very little phosphorus for urinary elimination. Likewise, a shortage of phosphorus for deposition with calcium in the bone was created, thus accounting for the increased elimination of urinary calcium. However, the excellent mineral balances indicate that the pathological disturbance of osteomalacia in this case was in the process of reparation, presumably under the influence of vitamin $D$ that was derived from the diet and medication administered in the pre-experimental period. Although there was no exact record as to how much vitamin D was supplied in the previous dietary, it is certain that a number of hens' eggs were given. It was also recorded that she received altogether $40 \mathrm{cc}$. of cod liver oil in four doses on two different dates. It may 
TABLE II

Case 1. S. C. C. Calcium and phosphorus metabolism during vitamin $D$ depletion, after the administration of small doses of vitamin $D$ and after supplementing basal diets with eggs

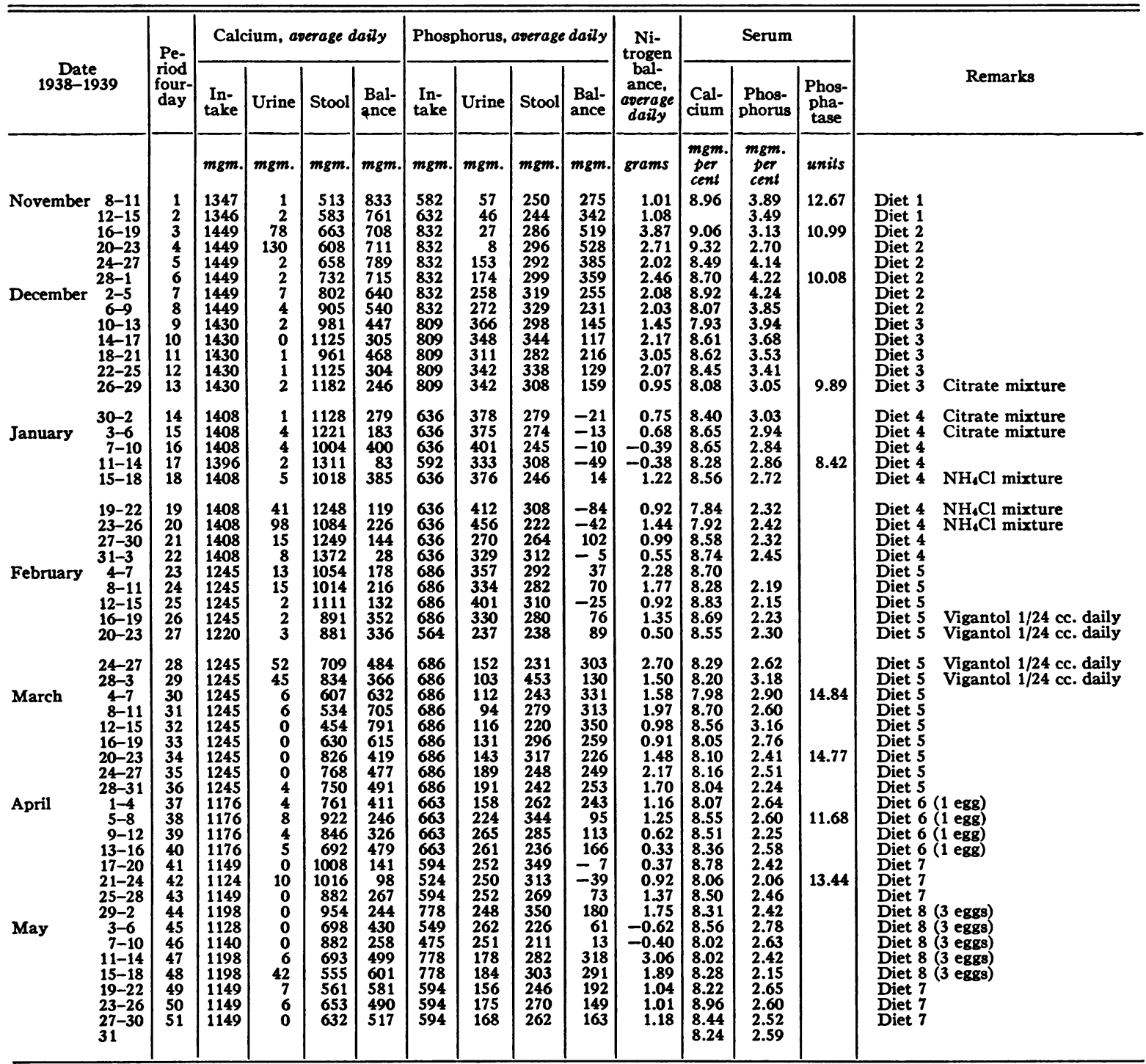

be surmised that before the present study she received some vitamin $D$ which, though small in amount, was sufficient to bring about the favorable calcium and phosphorus retention observed at the beginning of the experiment.

In order to see how long this degree of calcium and phosphorus retention would continue and to determine whether the experimental diets would maintain this favorable state of affairs, observation was extended for 12 periods, or almost seven weeks, without modification of the regimen. It is plainly seen that the urine calcium disappeared promptly and the fecal calcium increased progressively, so that by the end of period 12 it had become twice as much as that at the beginning, and the positive calcium balance was reduced to only 21 per cent of the intake. Simultaneously, the phosphorus balance was also reduced as the result of increased excretion both in the urine and in the stool, that in the former being more marked. There was a slight reduction of serum calcium and a more marked diminution of serum inorganic phosphorus. The initial drop of serum inorganic phosphorus in periods 1 to 


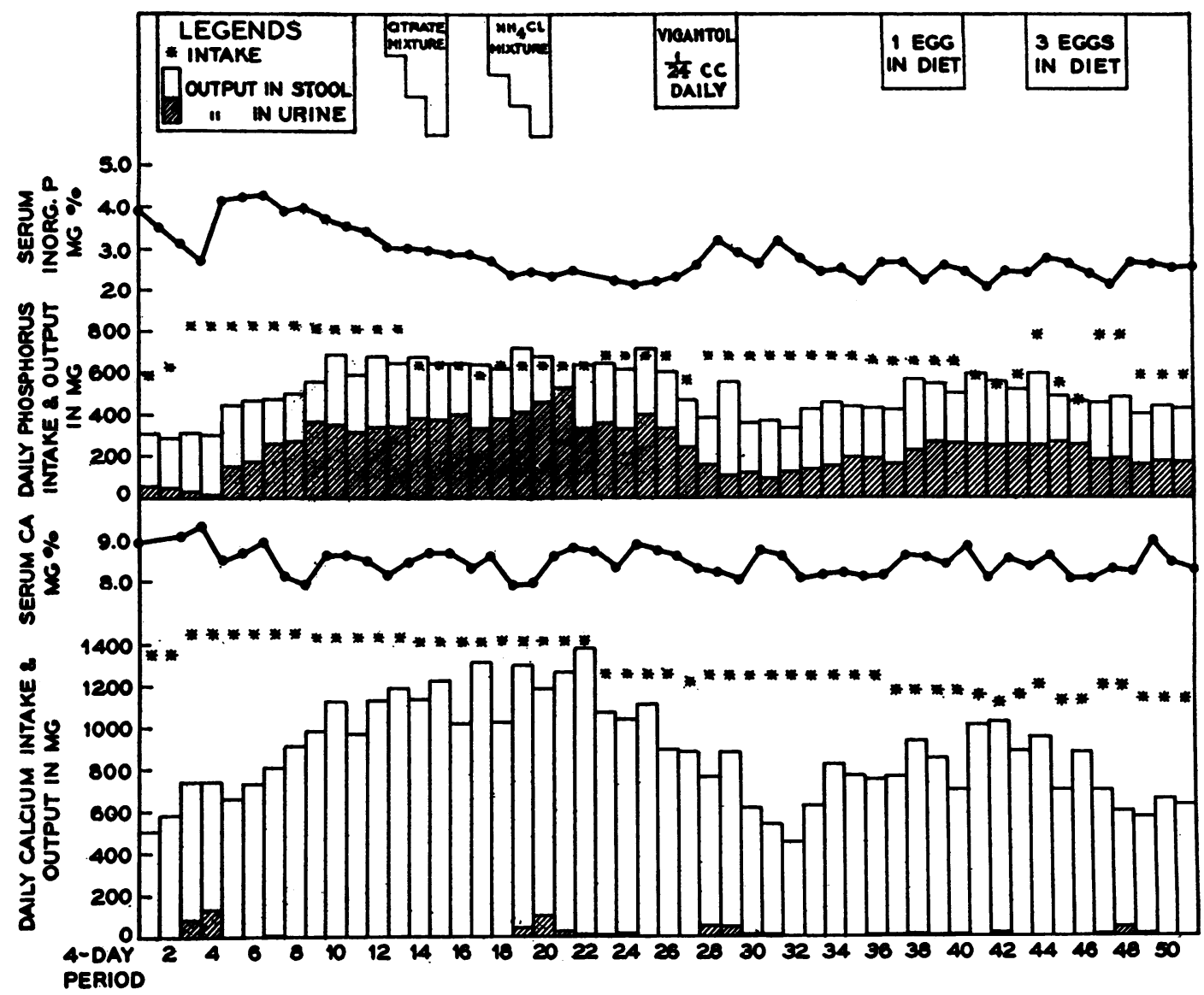

Fig. 1. Case 1, S. C. C. Calcium and Phosphorus Metabolism During Vitamin D Depletion, After the Administration of Small Doses of Vitamin D and After Supplementing Basal Diets With EgGs

4 was probably related to the relatively low phosphorus intake and the rapid deposition in the soft tissues and in the bones as the result of high nitrogen and calcium retention, while the subsequent decline was probably due to vitamin D depletion.

These data demonstrate clearly that the beneficial effect of the hospital diet on mineral metabolism, together with a few doses of cod liver oil, was real, and it lasted for approximately seven weeks. On the other hand, the experimental vitamin D-deficient diets were unable to maintain the good calcium and phosphorus balance initiated prior to their institution.

II. The effect of acid and alkali salt mixtures and extension of observations on vitamin $D$ depletion. We are not primarily concerned with the effect of acid and alkali in this part of the study. The data were included in the present communication merely for the sake of continuity. It may be noted, however, that daily administration of 200 to $400 \mathrm{cc}$. of a solution containing $0.6 \mathrm{~N}$ of sodium citrate and $0.9 \mathrm{~N}$ of citric acid during periods 13 to 15 and 60 to 180 milliequivalents of a mixture of ammonium chloride and ammonium carbonate during periods 18 to 20 did not affect the calcium and phosphorus metabolism in any important way. The urine, however, contained considerable amounts of calcium and an augmented quantity of phosphorus during the period of ammonium chloride mixture administration. These were expected from the acid effect. The calcium retention showed a further tendency to diminish, irrespective of the acid and alkali administration, so that by the end of period 22 the calcium retention became almost nil. The 
phosphorus balance was slightly negative during these periods because of the somewhat reduced intake, together with the poor calcium and nitrogen balance. The negative phosphorus balance was slightly exaggerated by the ammonium chloride mixture administration in periods 19 to 20 . The serum calcium fluctuated around $8.5 \mathrm{mgm}$. per cent but the inorganic phosphorus declined further to below $2.5 \mathrm{mgm}$. per cent, partly from reduction of phosphorus intake in the diet and partly from continued vitamin $\mathrm{D}$ depletion. We are, therefore, inclined to consider that, besides some minor changes, the data obtained during periods 13 to 22 represent an extension of the observation made in the first 12 periods. In other words, the calcium retention in the course of 22 periods on the experimental diets changed from 54 per cent of the intake to only 2 per cent. This is undoubtedly the result of vitamin $\mathrm{D}$ depletion.

III. The effect of a minimal dose of Vigantol. Vigantol was diluted with olive oil 1 to 24 and $1 \mathrm{cc}$. of this diluted solution containing 500 international units of vitamin $\mathrm{D}$ was given daily during periods 26 to 29 . The effect on the calcium and phosphorus metabolism was promptly shown up. The fecal calcium decreased rapidly and urinary calcium began to appear. The calcium retention, which averaged not more than 15 per cent of the intake in periods 23 to 25 just prior to Vigantol administration, increased progressively, reaching its maximum in period 32 , twelve days after Vigantol was discontinued. The calcium retention then represented almost 64 per cent of the intake. The phosphorus retention ran a parallel course, increasing from only 4 per cent of intake in periods 23 to 25 to 51 per cent in period 32. This was mainly the result of reduction of urine phosphorus. The serum calcium did not show any important change but the serum inorganic phosphorus was distinctly improved.

However, the beneficial effect of such a small dose of vitamin $\mathrm{D}$ for a limited period was obviously not a lasting one, as shown by the observations during periods 33 to 36 . The calcium retention definitely diminished and the same was true with the phosphorus balance. The serum inorganic phosphorus also declined. Time did not permit extension of the observation to the time when the calcium and phosphorus retention would resume the original low value.

IV. The effect of one and three hens' eggs. When one egg was added to the diet during periods 37 to 40 no definite effect could be discerned in the calcium and phosphorus metabolism. It is estimated that one egg yolk at its best contained no more than 60 international units of vitamin $D$, an amount which would be hardly detectable in human experiment. However, immediately after the single egg was withdrawn, the calcium retention was distinctly poorer in periods 41 to 43 and the fecal calcium reached the same original high level as that before Vigantol was administered. This may be taken to mean that the antirachitic potency of a single egg did help in checking the declining tendency of the calcium balance, although it was not sufficient to reverse the process.

When three eggs were included in the diet during periods 44 to 48 , unequivocal changes in the calcium and phosphorus metabolism were observed. The fecal calcium decreased with an increase of calcium retention from an average of 15 per cent in periods 41 to 43 to over 50 per cent of the intake in periods 48 to 49 . Appreciable amounts of calcium appeared in the urine in the last period of the egg ingestion. Toothache and excision of a radicular cyst disturbed the food intake, resulting in negative nitrogen balance and very poor phosphorus retention during period 45 to 46. However, subsequent observation showed definite improvement in phosphorus retention, due chiefly to reduction of urinary phosphorus. The serum calcium and inorganic phosphorus showed no significant changes. No attempt was made to determine the exact duration of the beneficial effect of the three-egg regimen.

\section{Case 2, L. C. Y., osteomalacia and rheumatic heart disease}

I. The exhibition of vitamin $D$ depletion on experimental vitamin $D$-deficient diet. This patient received $500 \mathrm{cc}$. of cod liver oil in June and just passed through the summer before the present investigation began in October. It is, therefore, not astonishing to see a positive calcium balance even when she was put on an intake of only $232 \mathrm{mgm}$. per day (periods 1 to 2 ). When the calcium intake was raised to over $1,200 \mathrm{mgm}$., 
TABLE III

Case 2. L. C. Y. Calcium and phosphorus metabolism during vitamin D depletion, after supplementing basal diets with eggs and after the administration of vitamin $D$ in minimal and full doses

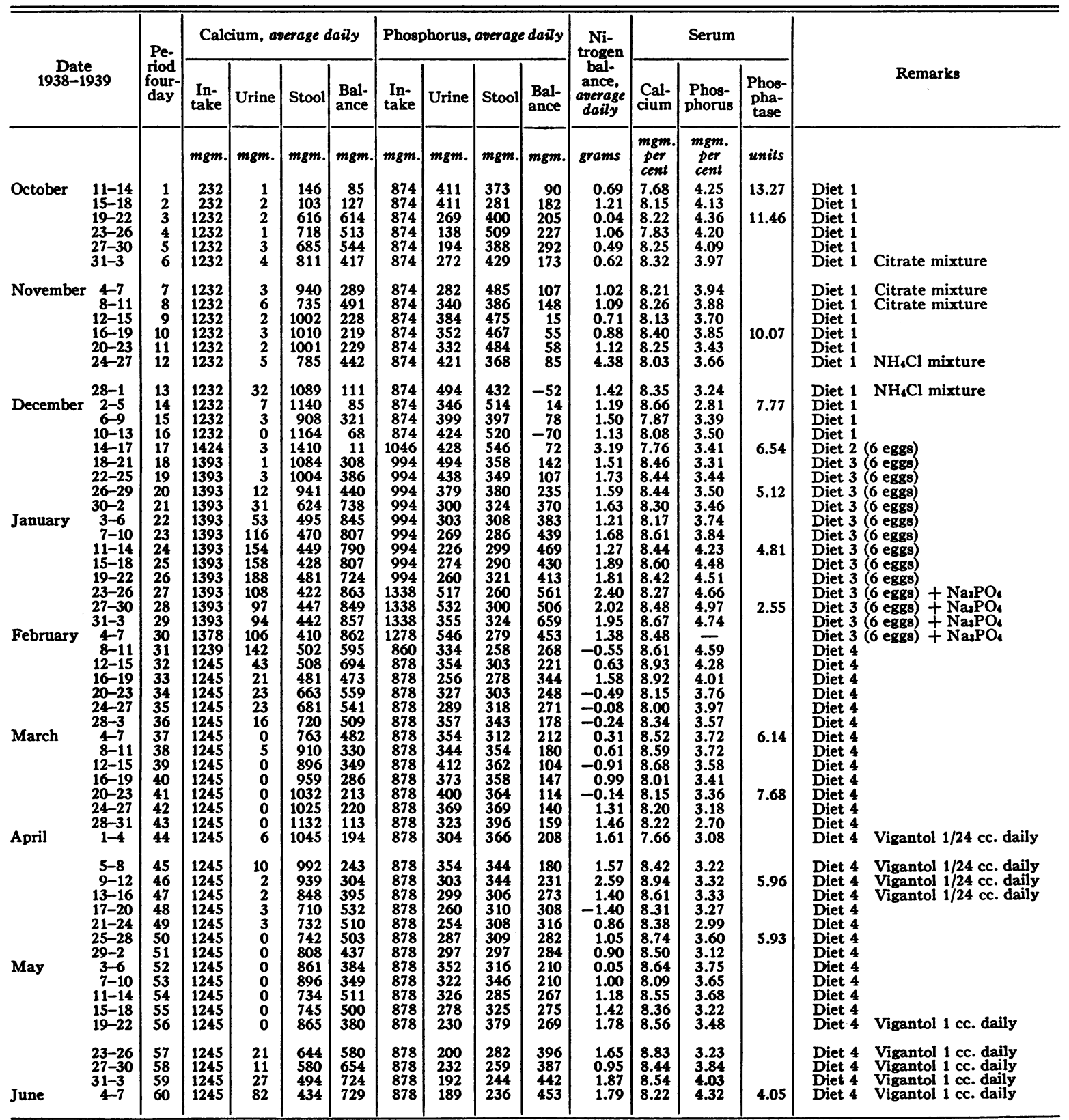

the average calcium retention in periods 3 to 5 amounted to 45 per cent of the intake. The average phosphorus retention of the corresponding periods was almost 28 per cent of the intake. The urinary phosphorus ran low. The serum calcium was around $8 \mathrm{mgm}$. per cent but the inorganic phosphorus was more than $4 \mathrm{mgm}$. per cent.
The effects of 100 to $300 \mathrm{cc}$. of a solution containing $0.6 \mathrm{~N}$ sodium citrate and $0.9 \mathrm{~N}$ citric acid and a solid mixture of 60 to 120 milliequivalents of ammonium carbonate and of ammonium chloride were studied during periods 6 to 8 and 12 to 13 , respectively. As in Case 1 , we cannot attribute any important changes to the use of these salt mixtures other than a slight drainage 


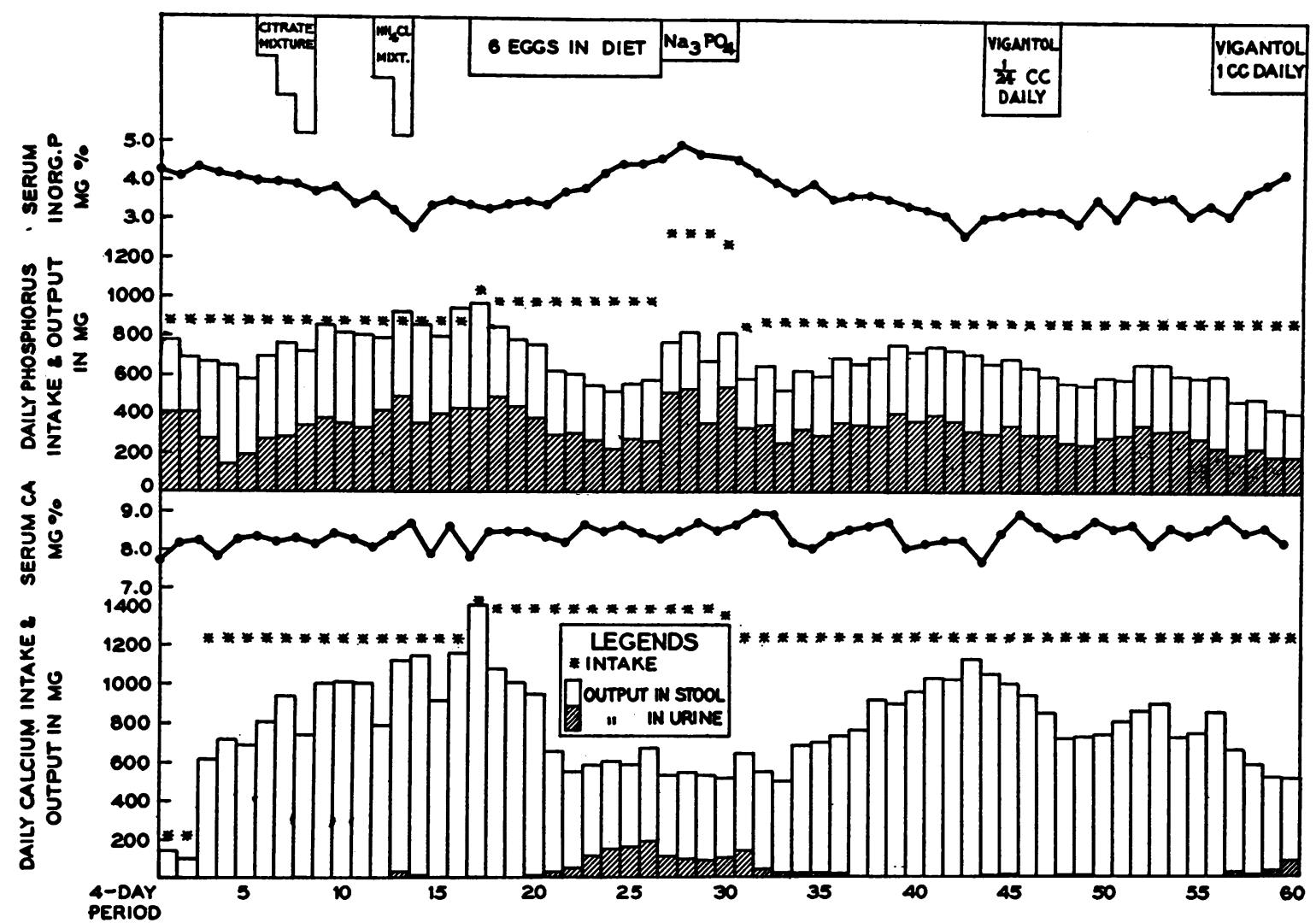

Fig. 2. Case 2, L. C. Y. Calcium and Phosphorus Metabolism During Vitamin D Depletion, After Supplementing Basal Diets With Eggs and After the Administration of Vitamin D in Minimal and Full Doses

of urinary calcium and a definite increase of urinary phosphorus, together with slight impairment of phosphorus retention during the periods of ammonium chloride administration.

It is, however, clear that throughout periods 3 to 16 there was an uninterrupted trend of reduction in both the calcium and the phosphorus retention. The calcium retention in period 16 measured less than 6 per cent of the intake. This was entirely due to a progressive increase in fecal calcium. Both the urine and the fecal phosphorus increased, the urine more so. The phosphorus balance during period 16 was definitely negative. The serum calcium did not change but the inorganic phosphorus dropped to a distinctly lower level. Again these findings may be taken as characteristic effects of vitamin D depletion. The experimental diets were sufficiently devoid of vitamin D to allow such depletion to take place.

II. The effect of six eggs. Two hundred grams of egg, approximately equivalent to six average sized Chinese hens' eggs, contained 80 grams of yolk, which was estimated to contain not more than 400 international units of vitamin D. This was given daily during periods 17 to 26 . There was a sudden progressive reduction of fecal calcium and a similar reduction of urinary and fecal phosphorus. With further reduction of fecal calcium, urinary calcium began to appear and reached a significant proportion by the end of the last period of egg ingestion. The result of these changes was a marked increase of calcium and phosphorus retention which reached their maximum-namely, about 61 per cent of the intake-in the sixth period of egg ingestion. This high degree of calcium retention was maintained for the following 4 periods. The changes in the serum calcium were insignificant but the inorganic phosphorus rose to a perfectly normal level.

When more phosphorus was given in the form of a solution of trisodium phosphate in periods 27 to 30 , the urinary calcium decreased slightly 
and there resulted a slight further increase of calcium retention. The phosphorus retention was also further increased but most of the excess phosphorus appeared in the urine.

The optimal calcium balance continued unchanged for about 7 periods after the egg ingestion was discontinued. Then the effect of the egg diet began to wear off. First of all the urine calcium decreased and later disappeared. The fecal calcium increased steadily. Both the urine and the fecal phosphorus increased. The serum calcium decreased slightly and the serum inorganic phosphorus once more came down to a very low level. By period 13 the effect of the egg diet may be considered to have entirely disappeared. The calcium retention then was only 9 per cent of the intake compared with the average optimal retention of 57 per cent during periods 22 to 26.

To summarize this experiment: six eggs daily for forty days brought about a maximal calcium and phosphorus retention in twenty-four days after the commencement of their ingestion. The maximal retention was maintained for forty-eight days or twenty-eight days after the egg ingestion was stopped. It took sixty-eight days after its discontinuation for the beneficial effect to disappear completely.

III. The effect of minimal and full doses of
Vigantol. One cc. of a diluted Vigantol (1:24) containing 500 international units of vitamin D was given during periods 44 to 47 . Its action on the calcium and phosphorus balances was seen immediately and the maximal effect was obtained in the period immediately after the drug was discontinued. This improved calcium and phosphorus retention continued for 3 more periods before it began to decline. A slight increase of the serum calcium and inorganic phosphorus was also noticeable. The definite but transient effect of the small amount of Vigantol observed in the present case resembled that observed in Case 1 in a remarkably close manner.

When the full dose of Vigantol, $1 \mathrm{cc}$. containing 12,000 international units of vitamin $\mathrm{D}$, was given in the last 5 periods, the calcium retention was naturally further improved and calcium began to appear in the urine. The urine and fecal phosphorus decreased, resulting in more favorable phosphorus balance. The serum inorganic phosphorus was raised to over $4 \mathrm{mgm}$. per cent. There was no time to observe the maximal degree of improvement and the course of depletion.

\section{Case 3, L. T. L., osteomalacia and rickets}

The vitamin $D$-deficient and calcium-low diet and the production of negative mineral balances as a sign of vitamin $D$ depletion. This patient

TABLE IV

Case 3. L. T. L. Calcium and phosphorus metabolism during vitamin $D$ depletion while on a low calcium diet

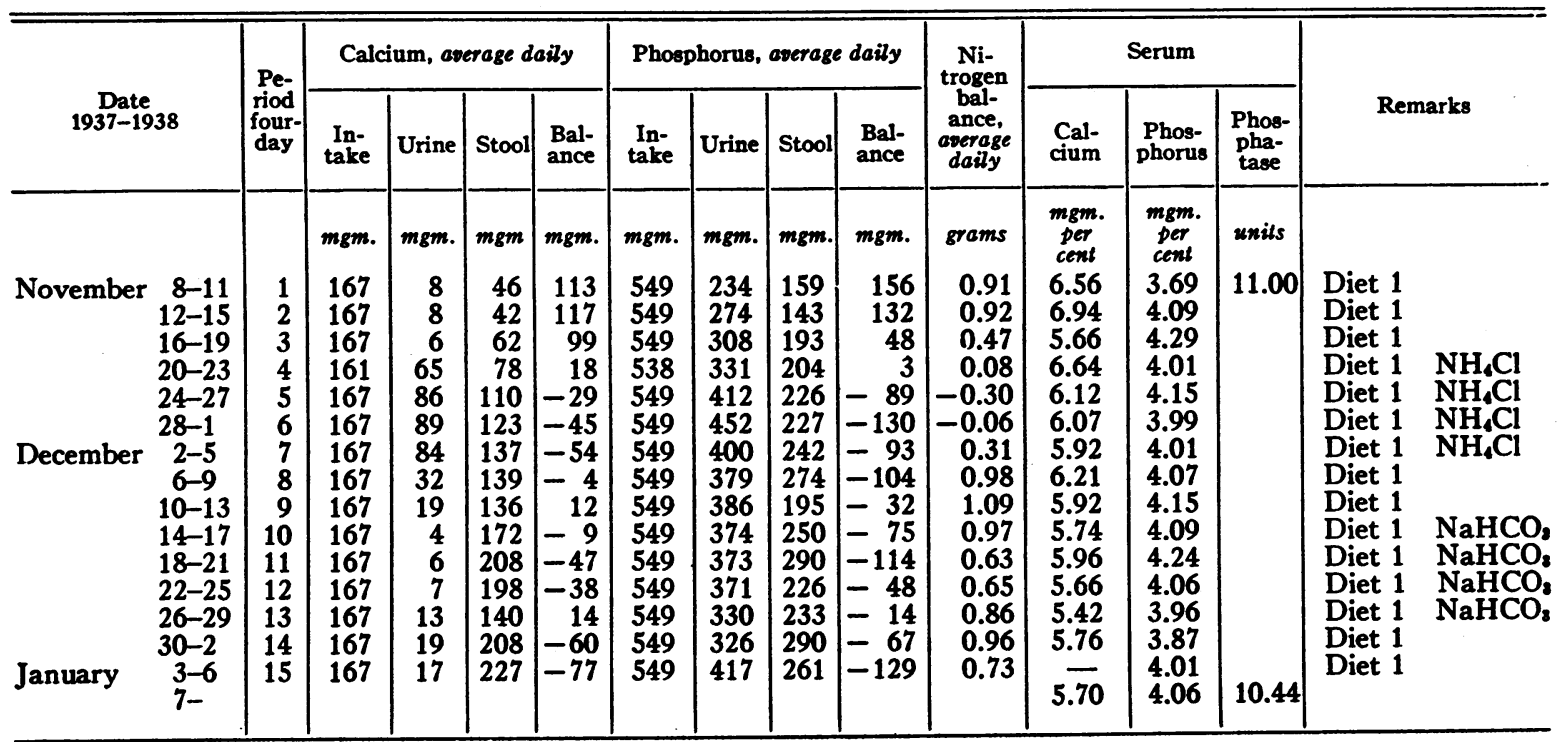




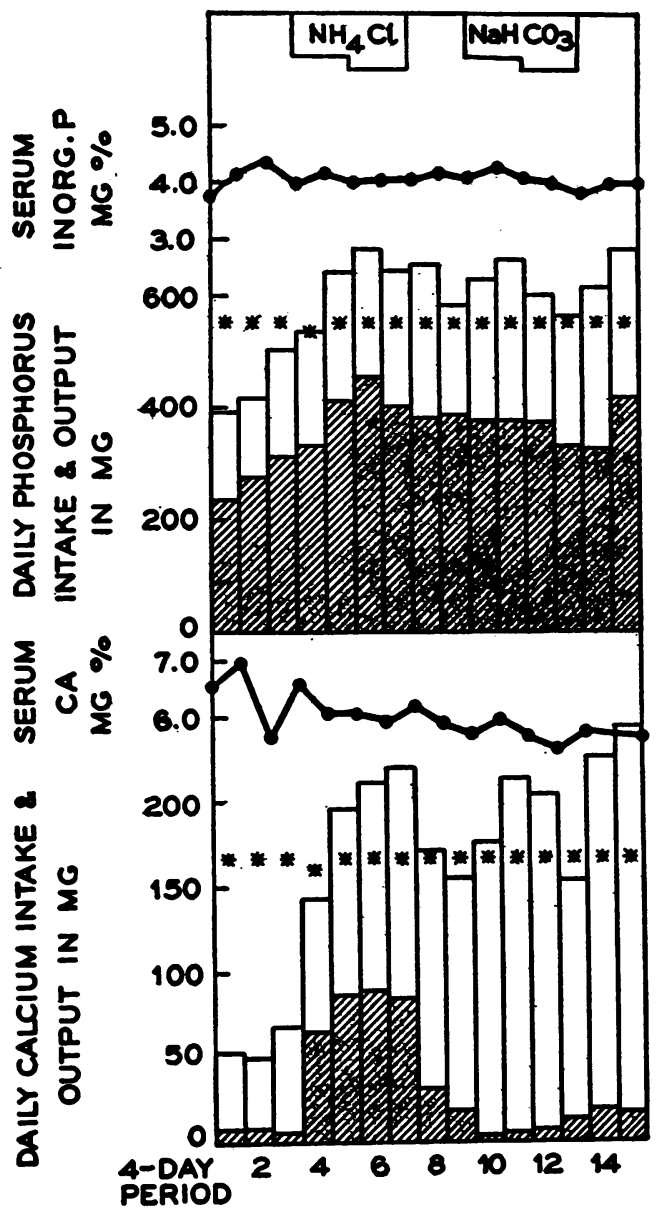

Fig. 3. Case 3, L. T. L. Calcium and Phosphorus Metabolism During Vitamin D Depletion While on a Low Calcium Diet

Legends as in other figures.

had been on a full hospital diet for one month in the orthopedic service prior to the present study. The diet included eggs, milk and sometimes liver. No vitamin $\mathrm{D}$ medication or ultraviolet irradiation was prescribed. When she was put on the low calcium and vitamin D-deficient diet, her calcium and phosphorus metabolism was found to be remarkably conservative. She was able to retain, on the average, $110 \mathrm{mgm}$. of calcium out of a daily intake of only $167 \mathrm{mgm}$. in periods 1 to 3. The phosphorus retention was equally favorable. The serum calcium, rather low to start with, showed a tendency to decline further with the low calcium intake. The serum inorganic phosphorus, however, was maintained in the neighborhood of $4 \mathrm{mgm}$. per cent.
Ammonium chloride and sodium bicarbonate in doses of 30 to 40 milliequivalents daily were given during periods 4 to 7 and 10 to 13 , respectively. The marked increase of urinary calcium and phosphorus was attributed to the action of the acid-producing salt, ammonium chloride, but no change could be assigned to sodium bicarbonate. This opinion was well corroborated by the findings of Farquharson and associates (6).

When one peruses the figures for fecal calcium, ignoring the urinary calcium for the time being, a staircase rise of fecal calcium throughout periods 4 to 15 becomes apparent. This progressive increase of fecal calcium was in no way related to the acid and alkali administration. The calcium balances during periods 10 to 15 , with the exception of period 13, became negative, mainly as the result of large stool calcium excretion, which exceeded the intake. The phosphorus balances were also negative, with the urine and stool sharing the drainage. The serum calcium dropped to below $5.5 \mathrm{mgm}$. per cent. All these changes are best explained on the basis of vitamin $\mathrm{D}$ depletion. The distinguishing features in this experiment are the low calcium intake and the negative calcium and phosphorus balances. These are the circumstances which must have existed in all cases of osteomalacia at one time or another, although they are rarely observed in metabolic experiments. The negative calcium and phosphorus balances observed in this patient on a vitamin D-deficient and calcium-low diet, although small in extent, are significant, particularly when they are continued. They give us an insight into the pathogenesis of osteomalacia.

\section{Case 4, Baby W. H. S., normal infant}

Incipient changes in calcium metabolism following vitamin $D$ depletion. This was a normal infant whose mother was suffering from osteomalacia and who had received $20 \mathrm{cc}$. of Vigantol in four days about one month before delivery and $30 \mathrm{cc}$. cod liver oil daily for six days immediately after parturition. The calcium and phosphorus metabolism of the mother was undoubtedly benefited by the vitamin $\mathrm{D}$ intake, and the baby was protected from fetal rickets. The experiment was primarily designed to demonstrate the maternal transmission of vitamin $\mathrm{D}$ in the milk, the details 


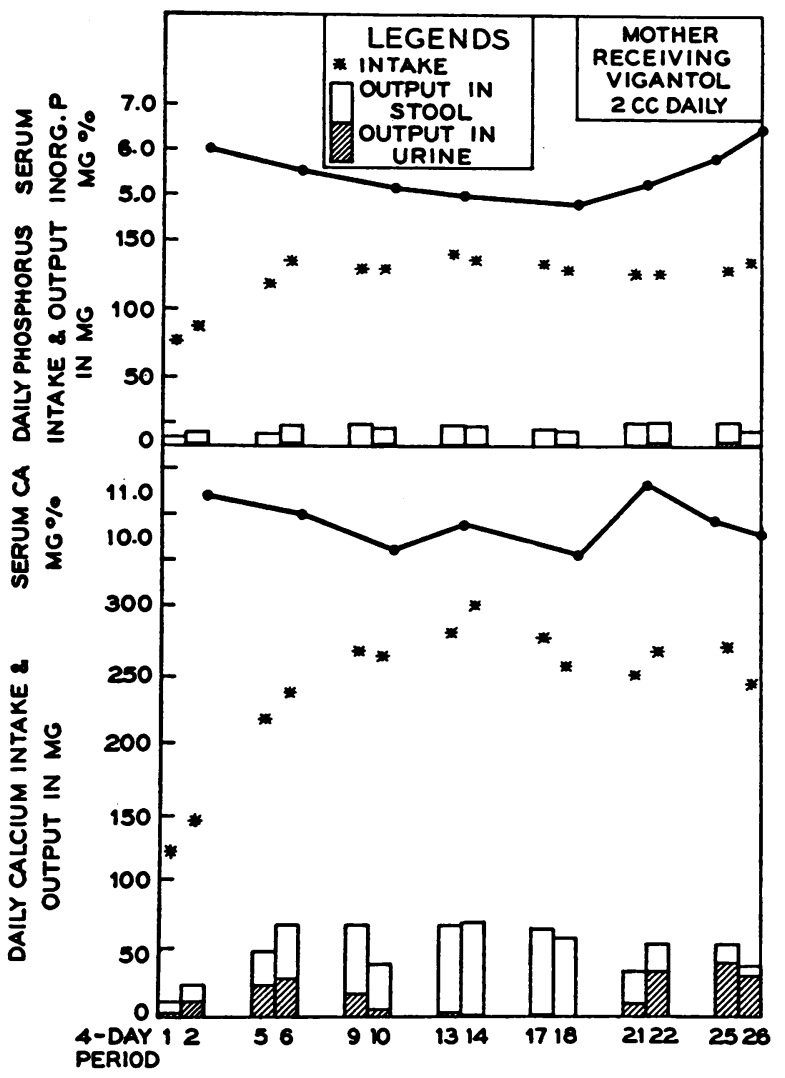

Fig. 4. Case 4, W. H. S. Calcium and Phosphorus Metabolism of a Breast-fed Infant Showing Vitamin D Depletion With Subsequent Replenishment, All Via Mother's Milk

of which are reported in a preceding communication (15)..$^{1}$ We are interested here particularly in the changes of calcium and phosphorus metabolism during the period of vitamin $\mathrm{D}$ depletion. The baby received mother's milk exclusively throughout the experiment and the mother was not given any vitamin D until 18 periods of control observation were completed in seventy-two days. A series of events happened during these periods (Figure 4). The urine calcium, which was relatively large in amount, disappeared slowly. The fecal calcium increased but a good calcium retention was still maintained. There was a slight lowering of serum calcium and inorganic phosphorus. However, no significant changes were observed in the phosphorus balances which remained conservative.

\footnotetext{
1 Part of Figure 4 of the preceding paper (15) is reproduced here for convenience.
}

When 2 cc. of Vigantol were given to the mother daily immediately after period 18 and continued till the end of the experiment, an undetermined amount of vitamin $D$ must have reached the baby through the milk. The reverse phenomenon took place. The fecal calcium decreased almost to nil. Urinary calcium made its appearance again. The net percentage of calcium retention was slightly better. The serum calcium and inorganic phosphorus showed definite increase. The phosphorus balance remained as good as before.

The changes in the calcium and phosphorus metabolism observed in the first 18 periods (actually 10 periods), may be representative of vitamin $\mathrm{D}$ depletion. Inasmuch as the mineral retention remained favorable, the shifting of calcium elimination from the urine to the stool might be regarded as an incipient change in the mineral metabolism in the direction of development of rickets or osteomalacia. This was corroborated by the suggestive $x$-ray findings shortly before vitamin $\mathrm{D}$ was given to the mother.

\section{DISCUSSION}

The results of the present investigation confirm completely our contention repeatedly expressed in former communications $(2,3,4,15)$ that the diet during the pre-experimental period and the vitamin $\mathrm{D}$ medication, even though in small doses and given some time previously, play an important rôle in determining the status of calcium and phosphorus metabolism at the beginning of the experiment. It cannot be doubted that small doses of vitamin $D$ in the form of cod liver oil given for a limited period and the even smaller amount of vitamin $\mathrm{D}$ included in the hospital diet for many weeks can bring about a favorable calcium and phosphorus balance. Although this beneficial effect is of limited duration it cannot be appreciated until one is prepared to extend control observation on a vitamin D-deficient diet for a prolonged period. This circumstance has not escaped the attention of early investigators. Hess, Weinstock and Tolstoi (10) realized the influence of the diet during the pre-experimental period on the susceptibility of rats to rickets. The refractory state occasionally encountered in certain groups of rats was found to be due to the 
liberal diet during the pre-experimental period. It was overcome and the young rats were rendered susceptible to rickets by feeding the mother and young a less adequate diet throughout the suckling period.

Thus in any investigation on calcium and phosphorus metabolism, the status of the vitamin $\mathrm{D}$ factor, past and present, must be clearly defined and segregated, lest false interpretations be made. This is not an easy matter, in view of the fact that minimal doses of vitamin $\mathrm{D}$ are effective and their effect is sustained long after their discontinuation. Therefore, in order to ascertain and separate the effect of previous vitamin D administration and of subsequent withdrawal, control metabolic observations for many periods are essential.

On the basis of the present study we feel justified in concluding that our generally-used experimental diets in this series of investigations are deficient in vitamin $\mathrm{D}$. They certainly cannot be relied upon to exert any curative effect on osteomalacia or rickets; nor can they maintain whatever beneficial effect may have accrued from a previous regimen. It must be noted, however, that these diets, essentially cereal-vegetable-meatoil, conform to the better one of the two types of dietary used by the mass of population in Peiping (8). Common vitamin D-containing foods, such as eggs, milk, butter, fish, chicken and liver, etc., are not available to the majority of the population. It is our impression that they derive their full or partial vitamin $D$ requirement from non-dietary source or ultraviolet irradiation from the sun. It is also our opinion that subclinical vitamin $\mathrm{D}$ deficiency must be rather prevalent among the population, particularly those who lead a confined life.

The most interesting and instructive feature in the present investigation is related to the metabolic changes following the withdrawal of vitamin $\mathrm{D}$. In a way these changes may be taken to represent the metabolic process in progress in the course of development of rickets or osteomalacia. In the case of calcium, these consist of (1) a diminution and, later, disappearance of urine calcium; (2) a progressive increase of stool calcium as the result of increasing difficulty in the intestinal absorption of the mineral; and (3) a progressive decrease of calcium retention on adequate intake or actual loss of the element on low intake. Simultaneously, or somewhat later, the phosphorus excretion is increased both in the urine and in the stool. The increased urine phosphorus presumably represents that portion of the absorbed phosphorus which fails to be deposited in the bones because of lack of absorbed calcium. The fecal phosphorus is increased simply by reason of the increased unabsorbed calcium in the bowels. Likewise, phosphorus balance suffers. Both serum calcium and phosphorus decrease but one may be more seriously or sooner affected than the other.

In spite of the fact that the derangements of calcium and phosphorus metabolism in advanced vitamin $\mathrm{D}$ deficiency are well known and that the metabolic response to vitamin $\mathrm{D}$ therapy in such cases is familiar to the students of rickets and osteomalacia, the literature is devoid of any information on the incipient changes that occur in the pre- or subclinical stage of the disease. Skaar's (18) investigation on experimental rickets in dogs was perhaps the only published work which included metabolic observations in the course of development of rickets. Even in this work the author failed to realize the importance of publishing data on the changes in the paths of excretion of calcium and phosphorus which accompanied the poor retention of the minerals.

The experience gained from the study of the effect of small doses of vitamin $D$ on the calcium and phosphorus metabolism in osteomalacia opens up the possibility of assaying in human beings the relative antirachitic potency of different vitamin D-containing foods. It would be of still greater interest to compare the antirachitic potency of vitamin Ds of different origin, a question which has not been answered satisfactorily by clinical experiences with rickets $(1,5,13,14)$.

Heymann $(11,12)$ demonstrated the presence of vitamin $\mathrm{D}$ in the liver and the blood plasma of rabbits for as long as twelve weeks after a single dose of viosterol equivalent to 200,000 international units. This was more or less confirmed by Guerrant and associates (7) in growing calves and by Vollmer (19) in human beings. These facts about the storage of vitamin $D$ following oral administration provide a logical explanation for the prolonged duration of the antirachitic effect of vitamin $\mathrm{D}$. More work must be done, however, to determine the extent of storage 
in relation to the size of the dose administered. Further work along the lines started in the present investigation would also answer the question as to whether the duration of the antirachitic effect of a given dose of vitamin $\mathrm{D}$ would be proportional to the size of the dose.

\section{SUM MARY}

Calcium and phosphorus metabolism was studied in 3 patients with osteomalacia and 1 normal infant born of an osteomalacic mother. Prolonged observation was made while the patients were on vitamin $\mathrm{D}$-deficient diets. The infant was given breast milk exclusively, while the mother was on a similar vitamin $\mathrm{D}$-deficient diet. At the beginning all 4 cases showed conservative calcium and phosphorus balances, due presumably to prior vitamin D store either from the hospital diet or from medication as cod liver oil. But in the course of time the following metabolic changes were observed. The urine calcium decreased and then disappeared; the fecal calcium became progressively increased; and the calcium balance was reduced or became negative when the calcium intake was low. The phosphorus metabolism followed a similar unfavorable course; both the urine and the fecal phosphorus were increased. The serum calcium and inorganic phosphorus usually diminished but slightly in the course of these observations.

These metabolic changes were interpreted as evidence of vitamin $\mathrm{D}$ depletion. When they were put on the experimental diets, which were sufficiently devoid of vitamin $D$ to allow the depletion of the prior store, whatever beneficial effect they derived from the previous regimen disappeared in the course of time, giving place to all the metabolic changes enumerated above. These biochemical alterations are regarded as early signs of vitamin $\mathrm{D}$ depletion, as they must go on for some time before clinical evidence of such deficiency can be elicited. They are helpful in providing material for reconstructing the metabolic processes that underlie the development of rickets and osteomalacia.

Experiments with very small doses of vitamin $D$ in the form of Vigantol or the addition of eggs to the basal diet, as in Cases 1 and 2, demonstrated the efficacy of such minimal doses of vita- min $\mathrm{D}$, and confirmed the supposition that the favorable metabolic behavior observed at the beginning of the experiments in all the cases was due to previous vitamin $\mathrm{D}$ store which was given up very slowly.

\section{CASE ABSTRACTS}

Case 1, S. C. C., Hospital Number 64728, a Chinese married woman of 18 , was admitted to the obstetrical service on October 13,1938, after she had been in labor for thirty hours. She gave a history of pain in the thighs and difficulty in walking for about three and a half years. She had been married at the age of 14 . Her menstruation started shortly after marriage and she became pregnant for the first time just after the first period. She was apparently well in the first trimester of the pregnancy but she began to have pain in the thighs and difficulty in walking during the remaining course of pregnancy. She gave birth to the first child without dystocia in October 1935 . Her symptoms subsided for three months after parturition but recurred afterwards. The child was fed on the breasts. Pain in the thighs and difficulty in walking continued till April 1936 when her child died of diarrhea. The same symptoms were present from January to April 1937. The present pregnancy, her second one, commenced in January 1938, together with recurrence of pain in the thighs. The pain was noticeable on walking, which was difficult. She was confined in bed most of the time. There were no symptoms of tetany. Labor pains started approximately thirty hours before admission but no progress was made after repeated examinations by ignorant midwives. She was finally referred to this hospital for prolonged labor. She had productive cough for twenty days in 1937 and again for two weeks last spring. She never had any fever or hemoptysis. The rest of the past history was unimportant. Her diet was always poor, consisting of millet, mixed flour and salted turnip. It was roughly estimated that she got not more than 2,000 calories a day. During the present pregnancy her food intake was even further reduced.

Physical examination on admission revealed that the patient was very small in stature and undernourished. Her weight was $34.1 \mathrm{kgm}$. She preferred to lie on her side with the lower extremities drawn up and adducted. She complained of pain when her legs were extended and abducted. Gradually, full extension could be accomplished. The adductor muscles of the thighs were spastic and tender. Thoracic cage was asymmetrical with some tenderness over the ribs. There was slight degree of lordosis of the spine, intrusion of both acetabulae and slight knock-knee. Her head organs were normal except for pyorrhea alveolaris. The lungs were clear and the heart of normal size but rapid, with a basal systolic murmur. Blood pressure was 104/70. The abdomen was distended by gravid uterus which was in constant contraction. The fetal heart was irregular. Pelvic measurements were I.S. $21 \mathrm{~cm}$., I.C. $23.5 \mathrm{~cm}$., E.C. $17.5 \mathrm{~cm}$., and T.O. $8 \mathrm{~cm}$. Rectal and vaginal examinations showed 
that the cervical os was fully dilated, the membrane was intact and the fetal head was floating. There was slight pitting edema of the legs. The tendon reflexes were normal and Chvostek's and Trousseau's signs were negative.

Laboratory findings on admission showed that her urine contained some albumin, many white blood cells and occasional red blood cells. Phenolsulphonephthalein excretion was 65 per cent in two hours. Blood count: Hemoglobin 11.9 grams, red blood cells 4,040,000 and white blood cells 15,950 with 85 per cent polymorphonuclear leukocytes. Stool was positive for ova of Ascaris. Blood Wassermann test was negative. Serum calcium was $8.00 \mathrm{mgm}$. per cent, inorganic phosphorus $1.90 \mathrm{mgm}$. per cent and phosphatase 10.50 Bodansky units.

After admission the amniotic membrane ruptured and the umbilical cord prolapsed. The fetal heart stopped before the cord could be reduced. The dead fetus was finally delivered by craniotomy. The patient ran a lowgrade fever in the first week after parturition. Then the temperature went up higher, reaching over $39^{\circ} \mathrm{C}$. in the next two weeks. During this febrile period the patient did not have much complaint besides slight cough. There was slight dullness in the left upper chest anteriorly and dullness and many crepitant rales in the right lower interscapular area. Blood and urine cultures were sterile. Sputum contained neither pneumococci nor tubercle bacilli. X-ray of chest revealed shadows suggestive of advanced pulmonary tuberculosis of right lung and moderate involvement of left side. X-ray of the bones at the same time showed slight to moderate degree of osteoporosis of all the bones, triradiate deformity and contraction of the pelvis, old pathological fractures in the pubic and ischial bones, right radius and ulna and a few of the ribs. The urine then contained but a faint trace of albumin. There was a leukocytosis of 11,000 to 25,000 . No parasite was ever found in the blood smear. With supportive treatment the fever disappeared in one month. The general condition gradually improved and the physical and $x$-ray findings in the chest also improved. The urine became clear and the leukocytosis disappeared. Through the courtesy of Dr. K. T. Lim of the obstetric service, the patient was transferred to the metabolism ward for study on November 7, 1938.

During the postpartum period the patient was given a soft diet and later a high caloric soft diet. These diets, including animal protein and eggs, were supposed to be adequate. She received two doses of cod liver oil, 10 cc. each, on October 17, 1938, and another two similar doses on November 4, 1938. Throughout the period of study in the metabolism ward the patient did well. However, her appetite was limited so that the quantitative diets were of small caloric value. In periods 45 to 46 she developed a swelling of the left cheek and toothache. This was diagnosed as a radicular cyst with infection. Excision was done. This disturbed the metabolic study for 2 periods because the food intake was irregular. The pain in the thighs disappeared gradually after hospitalization. The $x$-ray findings in the lungs also disappeared. She was discharged on June 1, 1939, in good condition.

Case 2, L. C. Y., Hospital Number 64677, a 45-yearold Chinese widow, was admitted to this hospital on October 6, 1938, with the complaint of multiple joint pain and muscular aching for more than two years. The patient began to have aching in the lower back and thighs in February 1933. These symptoms continued till September and then subsided. In March 1936 she developed pain in the left lower chest, cough and fever. While the latter symptoms continued, pain in the back and the thighs returned in October 1936. The pain gradually involved the whole lower extremities so that finally she could not walk. These symptoms became worse through the winter of 1937 when she had, in addition, numbness of the hands and feet and, on several occasions, spasms of the hands. She came to the outpatient clinic for treatment in June 1938. Rheumatic heart disease, with mitral stenosis but without heart failure, and osteomalacia were diagnosed. Thirty cc. of cod liver oil daily were prescribed and $500 \mathrm{cc}$. of the oil were supplied. At the same time edema of lower extremities was noticed for twenty days. However, the pain in the back and lower extremities was much improved following the medication. She was then able to walk with a stick.

She had had occasional attacks of palpitation of the heart and shortness of breath ever since she was 20 years of age. She gave no definite history of joint pains before the onset of the present illness. She had had attacks of epistaxis since the age of 15 or 16 . She had gradually lost her sense of smell in the year preceding her admission. Her menstruation had always been irregular and scanty and it had stopped two years previously. She had been married at 18 , but had never become pregnant. Her husband had died ten years previously of chronic cough and hemoptysis of many years' standing. Her diet had been poor, consisting of millet, white flour and corn flour, with salted and fresh vegetables. She had never had any animal food nor any fat or oil for cooking.

Physical examination revealed that the patient was short and undernourished. Weight was $36.8 \mathrm{kgm}$. and height was $148 \mathrm{~cm}$. She appeared comfortable. Her breath was foul. Post-pharyngeal mucosa and nasal mucosa were dry and atrophic. Many teeth were missing, a few were carious, and some were loose. There was pyorrhea alveolaris. The right lobe of the thyroid gland was slightly enlarged. The lungs were clear. The heart was slightly enlarged. A rumbling diastolic murmur was present at the apex. P-2 was not accentuated. Blood pressure was $96 / 60$. The abdomen was soft and no viscera were palpable. There was slight edema in the legs. No skeletal deformity or any bone tenderness was observed. No spasm or tenderness was present in the adductor muscles of the thighs. Chvostek's sign was positive but Trousseau's sign was negative. Tendon reflexes were normal. Pelvic measurements were normal. 
Laboratory findings. Urine was clear and P.S.P. excretion was 70 per cent in two hours. Blood count: Hemoglobin 11.9 grams, red blood cells 4,220,000, white blood cells 7,000. Blood smear was normal. Stool was normal. Blood Wassermann was negative. Serum calcium was $7.68 \mathrm{mgm}$. per cent, inorganic phosphorus 4.25 mgm. per cent and phosphatase 13.27 units. Plasma albumin was 3.47 per cent and globulin 3.45 per cent. Basal metabolic rate was +8 per cent. Electrocardiogram revealed normal mechanism. X-ray examination showed that the frontal area of the heart was 16 per cent oversized, with enlargement in the left auricular region. There was no gross deformity of the bones but moderate degree of osteoporosis and coarsening of trabeculae of all the bones were present. Old pathological fractures were seen in the right 5th and 9th ribs, and the left 6 th, 7 th and 10 th ribs. Old fractures were also present in both scapulae and in the left 2nd metacarpal bone. There was mild biconcavity of the lower thoracic and upper lumbar vertebral bodies and slight deviation of the symphysis pubis towards the right side.

In the course of study, which lasted from October 1938 to June 1939, the patient was much improved. She gained $13 \mathrm{kgm}$. of weight. Her cardiac condition was well compensated. Her atrophic rhinitis was also treated by means of estrone spray with improvement. She was discharged on June 10, 1939 in good condition.

Case 3, L. T. L., Hospital Number 60504, a Chinese girl of 16, was admitted to the orthopedic service on September 28, 1937, with the complaint of pain in the knees for three years and progressive deformity of the lower extremities, spine and chest for one and a half years. The patient was apparently well up to about three years before admission when she began to experience a dull pain in both knee joints, particularly on motion. Symptoms continued but were not severe. In January 1936 the patient had scarlet fever which cleared up in seven days. In March 1936 she began to notice difficulty in extending her knees. One month later her hips also could not be fully extended. Walking became difficult and she was completely confined in bed. Deformity of the lower extremities became progressively worse so that on admission the lower extremities were permanently held at $90^{\circ}$ at the hips and at the knees. With the onset of deformity of the lower extremities she had dull pain in the upper spine and in the sternum. This was followed by a bulging deformity in her right upper back and protrusion of the sternum. She denied having had any symptoms of tetany. The patient had been born in a poor family. Hunger had been a common experience. She had had a fair amount of sunlight exposure before she had become incapacitated by her deformities but practically none during the past year and half. Menstruation had not yet started.

Examination showed marked underdevelopment and

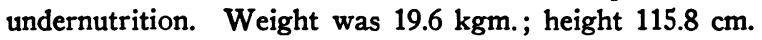
She was mentally alert but very quiet. No deformity of skull was present. Both wrists were distinctly enlarged. The thorax was markedly deformed with crumbling of the sternum, so that the distance between the sternal notch and the xiphoid process measured only $12 \mathrm{~cm}$. There were enlarged costochondral junctions over the lower ribs, obtuse subcostal angle and suggestive Harrison's groove. Marked thoraco-lumbar right kyphoscoliosis was present. The lower extremities showed marked muscular atrophy and contractures; extension at hips and knees was only possible to $90^{\circ}$. Pelvis was markedly contracted with very narrow pubic arch and the transverse outlet measured less than $3 \mathrm{~cm}$. Head organs were essentially normal. Skin was dry and hyperkeratotic. Dark adaptation was only $1 / 20$ of normal. Superficial lymph nodes were palpable. Thyroid gland was diffusely enlarged but more so on the right side. There were no signs of increased vascularity. Lungs showed dullness, with diminished breath sounds in the right upper area. The heart was normal. The abdomen protruded and the liver edge was palpable. Chvostek's sign was positive, but Trousseau's sign was negative and Erb's sign was doubtful.

Routine laboratory examination revealed normal urine, moderate anemia with slight eosinophilia and presence of ova of Taenia in the stool. Blood Wassermann test was negative. Serum calcium was $7.5 \mathrm{mgm}$. per cent, inorganic phosphorus $3.1 \mathrm{mgm}$. per cent and phosphatase 7.0 Bodansky units. Plasma albumin was 3.24 per cent and globulin 3.04 per cent. P.S.P. excretion and urea clearance were normal. Basal metabolic rate was +17.3 per cent. X-ray examination showed that all the bones were moderately to markedly osteoporotic. Marked scoliosis of the mid-thoracic spine and markedly exaggerated sacral curve were present. The vertebral bodies were moderately flattened with biconcave deformity. The pelvis was deformed with intrusion of both acetabulae. Costochondral ends of the ribs were broadened. The epiphyseal ends of radius and ulna were moderately expanded and hazy with faint areas of radiolucency. Irregularities and slight cupping were also present. Similar but less marked changes were observed in the epiphyseal ends of the tibia, fibula and femur. There was slight thickening of periosteum of most of the long bones. Her lungs appeared fairly clear.

The patient was first treated on the orthopedic service with Russell's traction to both lower extremities from September 28 to October 31, 1937. She was on full hospital diet; vitamin D medication was purposely withheld. With the kind permission of Drs. C. M. Meng and H. C. Fang, the patient was transferred to the metabolic ward for study on November 1, 1937, when her deformities of the lower extremities were considerably improved as the result of traction. Her taeniasis was treated successfully with aspidii oleoresina and proved to be due to Taenia solium. Three series of metabolic studies were made, the first of which was reported in the present communication. She was treated, after the first series of study, with ultraviolet irradiation and improved greatly. However, she developed a psychosis which did not improve until some time after discharge on May 30, 1938.

Case 4; W. H. S., Hospital Number 66085, a Chinese male baby, was born of an osteomalacic mother on Feb- 
ruary 7,1939 , by cesarean section. The history was given under Case $4 \mathrm{~b}$ of the preceding paper (15).

\section{BIBLIOGRAPHY}

1. Bischoff, $H$., and Brieger, $H .$, Zur Frage der Wirkung von Vitamin $D_{2}$ und $D_{3}$ auf Rachitis und Spasmophilie. Klin. Wchnschr., 1938, 17, 1795.

2. Chu, H. I., Chou, S. K., Chen, K. C., Wang, S. H., Liu, S. H., and Hannon, R. R., Calcium and phosphorus metabolism in osteomalacia. IV. Report of an unusual case in a male with acute parathormone poisoning. Chinese Med. J., 1936, 50, 1.

3. Chu, H. I., Yu, T. F., Chang, K. P., and Liu, W. T., Calcium and phosphorus metabolism in osteomalacia. VII. The effect of ultraviolet irradiation from mercury vapor quartz lamp and sunlight. Chinese Med. J., 1939, 55, 93.

4. Chu, H. I., Yu, T. F., and Liu, W. T., Calcium and phosphorus metabolism in osteomalacia. VIII. The effects of ingestion of acid and alkali in patients with and without chronic nephritis. Chinese J. Physiol., 1939, 14, 117.

5. Drake, T. G. H., Comparison of the antirachitic effects on human beings of vitamin $D$ from different sources. Am. J. Dis. Child., 1937, 53, 754.

6. Farquharson, R. F., Salter, W. T., Tibbetts, D. M., and Aub, J. C., Studies of calcium and phosphorus metabolism. XII. The effect of the ingestion of acid producing substances. J. Clin. Invest., 1931, 10, 221.

7. Guerrant, N. B., Morck, R. A., Bechdel, S. L., and Hilston, N. W., Storage of vitamin D in the tissues of growing calves. Proc. Soc. Exp. Biol. and Med., 1938, 38, 827.

8. Guy, R. A., and Yeh, K. S., Peking diets. Chinese Med. J., 1938, 54, 201.

9. Hannon, R. R., Liu, S. H., Chu, H. I., Wang, S. H., Chen, K. C., and Chou, S. K., Calcium and phosphorus metabolism in osteomalacia. I. The effect of vitamin $D$ and its apparent duration. Chinese Med. J., 1934, 48, 623.

10. Hess, A. F., Weinstock, M., and Tolstoi, E., The influence of the diet during the pre-experimental period on the susceptibility of rats to rickets. J. Biol. Chem., 1923, 57, 731.

11. Heymann, W., Studies on the mode of action and the metabolism of vitamin D. J. Paediat., 1936, $8,480$.

12. Heymann, W., Metabolism and mode of action of vitamin D. II. Storage of vitamin D in different tissues in vivo. J. Biol. Chem., 1937, 118, 371.

13. Jacoby, G., Uber Rachitisbehandlung mit Vitamin $D_{2}-$ und $D_{3}-$ Konzentrat. Klin. Wchnschr., 1938, $17,1173$.

14. Lewis, J. M., Comparative antirachitic effectiveness of viosterol, cod liver oil, and percomorph liver oil. J. Paediat., 1937, 10, 155.

15. Liu, S. H., Chu, H. I., Su, C. C., Yu, T. F., and Cheng, T. Y., Calcium and phosphorus metabolism in osteomalacia. IX. Metabolic behavior of infants fed on breast milk from mothers showing various states of vitamin D nutrition. J. Clin. Invest., 1940, 19, 327.

16. Liu, S. H., Hannon, R. R., Chou, S. K., Chen, K. C., Chu, H. I., and Wang, S. H., Calcium and phosphorus metabolism in osteomalacia. III. The effects of varying levels and ratios of intake of calcium to phosphorus on their serum levels, paths of excretion and balances. Chinese J. Physiol., 1935, 9, 101.

17. Shohl, A. T., The effect of the acid-base content of the diet upon the production and cure of rickets with special reference to citrate. J. Nutrition, 1937, 14, 69.

18. Skaar, T., Experimental rickets. Studies in respect of calcium, phosphorus and magnesium metabolism. Acta Paediat., Supplement 1, 1931, 12, 1.

19. Vollmer, H., Distribution of vitamin $D$ in body after administration of massive doses. Am. J. Dis. Child., 1939, 57, 343. 